\title{
Article \\ Distributed Adaptive Formation Tracking Control under Fixed and Switching Topologies: Application on General Linear Multi-Agent Systems
}

\author{
Tianhao Sun ${ }^{1}$, Huiying Liu ${ }^{1}$, Yongming Yao ${ }^{1,2, *} \mathbb{C}$, Tianyu Li ${ }^{1}\left(\mathbb{D}\right.$ and Zhibo Cheng ${ }^{1}$ \\ 1 School of Mechanical and Aerospace Engineering, Jilin University, Changchun 130022, China; \\ sunth19@mails.jlu.edu.cn (T.S.); huiying20@mails.jlu.edu.cn (H.L.); litianyu@jlu.edu.cn (T.L.); \\ chengzb19@mails.jlu.edu.cn (Z.C.) \\ 2 State Key Laboratory of Superhard Materials, Jilin University, Changchun 130022, China \\ * Correspondence: ymyao@jlu.edu.cn; Tel.: +86-158-4401-0057
}

Citation: Sun, T.; Liu, H.; Yao, Y.; Li, T.; Cheng, Z. Distributed Adaptive Formation Tracking Control under Fixed and Switching Topologies: Application on General Linear Multi-Agent Systems. Symmetry 2021, 13, 941 . https://doi.org/10.3390/ sym13060941

Academic Editor: Vladik Kreinovich

Received: 8 April 2021

Accepted: 24 May 2021

Published: 26 May 2021

Publisher's Note: MDPI stays neutral with regard to jurisdictional claims in published maps and institutional affiliations.

Copyright: (c) 2021 by the authors. Licensee MDPI, Basel, Switzerland. This article is an open access article distributed under the terms and conditions of the Creative Commons Attribution (CC BY) license (https:// creativecommons.org/licenses/by/ $4.0 /)$.

\begin{abstract}
In this paper, the time-varying formation tracking problem of the general linear multi-agent system is discussed. A distributed formation tracking protocol based on Riccati inequalities with adaptive coupling weights among the follower agents and the leader agent is designed for a leaderfollowing multi-agent system under fixed and switching topologies. The formation configuration involved in this paper is expressed as a bounded piecewise continuously differentiable vector function. The follower agents will achieve the desired formation tracking trajectory of the leader. In traditional static protocols, the coupling weights depend on the communication topology and is a constant. However, in this paper, the coupling weights are updated by the state errors among the neighboring agents. Moreover, the stability analysis of the MAS under switching topology is presented, and proves that the followers also could achieve pre-specified time-varying formation, if the communication graph is jointly connected. Two numerical simulations indicate the capabilities of the algorithms.
\end{abstract}

Keywords: time-varying formation; adaptive control; multi-agent system; switching interaction topologies; leader-following tracking

\section{Introduction}

The research of distributed cooperation and coordination of MAS has received considerable attention in recent years due to the rapid development of computer science and the broad potential application prospects in various fields, such as cooperative surveillance [1], formation control of unmanned aerial vehicles [2], attitude synchronization of spacecrafts [3], swarm intelligence [4] and so on. The theoretical study of this problem involves many branches, including consensus, containment, synchronization and formation [5-7]. Formation control, as one of the critical problems in distributed cooperative control systems, aims to design control laws which could drive the states or outputs of all agents to reach a predefined configuration.

In the past few decades, many classical approaches have been proposed to achieve formation control, such as leader-follower, virtual structure and behavior-based approaches. In [8], Consolini studied the leader-follower formation control for mobile robots and the input constraints were considered. The virtual structure method was used to realize formation control and obstacle avoidance for a group of wheeled mobile robots under severe disturbances and system uncertainties in [9]. In [10], Lee proposed a decentralized behavior-based formation control algorithm and the function of obstacle avoidance was considered.

Although the above three traditional methods have proved very effective to solve the formation problem, it must be pointed out that these three strategies have their own 
weaknesses. For example, if the leader-follower based method lacks robustness due to the failure of the explicit leader, this may destroy the whole formation tracking, and the virtual structure-based method is not fully distributed as it requires each object to track its own waypoints [11].

Over the past few years, great progress has been achieved in consensus control of MAS, and numerous results have been acquired [12-14]. In [15], Ren extended the consensus algorithm for a second-order multi-agent system, and those algorithms were introduced to solve the formation problem. In the conclusion, Ren pointed out that "many existing methods, such as leader-follower, behavioral and virtual structure/virtual leader formation control approaches can be unified in the general framework of consensus building." More results on the formation control problem based on a consensus algorithm could be found in [16-18].

It should be pointed out that in [16-18], the formation controllers are both based on distributed static consensus protocol. In classical static consensus protocol, the designer should acquire the entire communication graph and compute the coupling weights between the neighbor agents. In other words, these consensus protocols cannot be computed and implemented by each agent in a fully distributed way. To address this limitation, Li [19] proposed an adaptive control method, which could be updated by the neighbor's information. In [20], Chu modified the protocol by adding a monotone increasing subitem to study the MAS's consensus problem with a directed communication graph. Not only for general linear systems, but for uncertain systems [21], nonlinear systems [22] and higher-order systems [23]; all of the above topics were addressed by previous researchers.

In practical application, the communication connection between agents will be affected by the environment and fault. When the working condition is poor or the agent fails, the communication signal may be interrupted. The addition of new agents will also cause a change in the connection relationship. When the communication topology changes over time, this makes the MAS more difficult to control. In the cooperative control of MAS, a very important problem is how to design the control protocol to drive the agents' synchronization based on the neighborhood information, in case of communication interruption, reconnection and communication delay. Hence, the research of a multi-agent system with switching interaction topologies will be more meaningful. In some previous studies, some preliminary results have been acquired for first-order consensus under switching topologies [24,25]. In [26], it was shown that if there is a spanning tree frequently in the union of each directed subgraph for first-order agents, then the consensus will be achieved asymptotically. In [27], Su studied the first-order leader-following consensus under an undirected switching communication graph. From the perspective of the formation problem, predecessors have also carried out some research. Mu proposed a distributed LQR consensus protocol for the quadrotors and two-wheeled mobile robots under a switching directed graph [28].

Compared with the previous relevant results, the contributions of this paper are as follows: First, the follower agents are not only required to achieve the predesigned timevarying formation; meanwhile, they should track the states of the leader agent. Compared with the time-invariant formation, the time-varying formation will bring the derivative of the formation information; this brings difficulty to design and analysis. The results for time-invariant formation tracking control cannot be directly applied to the time-varying formation problem. Second, the communication topology graph among the agents could be switching. The methods to deal with the fixed topology situations cannot be extended to the switching topologies situations. Third, in this paper, a distributed adaptive formation controller is proposed. The adaptive coupling weights could be updated by the information from the neighbors. This makes the strategy of control more flexible and precise.

The rest of this paper is organized as follows. In Section 2, some basic math concepts and problem formulation are introduced. The analysis for multi-agent fixed and switching systems is presented in Section 3. In Section 4, two numerical simulations are shown to verify the obtained results. Conclusions are drawn in Section 5. 


\section{Preliminaries and Problem Formulation}

In this section, some basic mathematical notation and concepts are introduced; after that, the problem will be presented.

\subsection{Basic Concepts of Algebraic Graph Theory and Relevant Notions}

Let $G=\{\mathcal{V}, \mathcal{E}\}$ describe an undirected graph, where $\mathcal{V}=\left\{v_{1}, v_{2}, \cdots v_{n}\right\}$ denotes the set of nodes. $\mathcal{E} \subseteq\left\{\left(v_{i}, v_{j}\right): v_{i}, v_{j} \in \mathcal{V} ; i \neq j\right\}$ denotes the set of edges, which belong to the graph. Define $\mathrm{A}=\left[a_{i j}\right] \in R^{N \times N}$ for the adjacency matrix; in addition, the elements of the weighted adjacency matrix are non-negative. An edge of $\mathrm{G}$ is denoted by $\varepsilon_{i j}=\left(v_{i}, v_{j}\right)$, representing the information flow from agent $i$ to agent $j$. The graph is called undirected if and only if the edges $\varepsilon_{i j}$ and $\varepsilon_{j i}$ are equivalent, which means that the information can be transmitted between them; on the contrary, we call a graph a directed graph if the edges $\varepsilon_{i j}$ and $\varepsilon_{j i}$ are different, and the flow of information has a direction. In this article, the graph is an undirected graph if there is no special statement. The elements of adjacency matrix $\mathrm{A}=\left\{a_{i j}\right\}$ satisfy that the edge $\varepsilon_{i j} \in \mathcal{E}$, then $a_{i j}=1$, otherwise $a_{i j}=0$. The degree matrix $\mathrm{D}=\operatorname{diag}\left(d_{1}, d_{2}, d_{3}, \cdots, d_{n}\right) \in \mathbb{R}^{N \times N}$ is a diagonal matrix where $d_{i}=\sum_{j=1}^{N} a_{i j}, N$ is the number of the follower agents. The Laplacian matrix of the graph $\mathrm{G}$ is defined as $\mathrm{L}=\mathrm{D}-\mathrm{A}$.

An undirected graph is said to be a connected graph if there is at least one path between any two nodes of the graph. For a leader-follower system, the leader agent can only send the information to its neighbor agents, but does not receive information from anyone. Furthermore, we define the leader adjacency matrix as $\mathrm{F}=\operatorname{diag}\left(b_{1}, b_{2}, b_{3}, \cdots, b_{n}\right) \in \mathbb{R}^{N \times N}$, corresponding to the adjacency matrix; the leader adjacency matrix is also a diagonal matrix. If the leader's information is available to the agent $\mathrm{i}, b_{i}=1$, and $b_{i}=0$, otherwise. Then, we have the definition of graph $\bar{G}$, which consists of the leader agent, the edges between the leader agent and its follower agents and the graph $\mathrm{G}$.

Lemma 1. The graph $G$ is connected if and only if Laplacian $L$ of $G$ has a simple zero eigenvalue.

Lemma 2. For a leader-following system, if the undirected graph $\bar{G}$ is connected, then the matrix $H=L+F$ is symmetric positive definite.

\subsection{Leader-Follower Formation Control Problem}

In this section, we consider such an MAS composed of $N+1$ agents. There is one leader agent and $\mathrm{N}$ follower agents in the system. The leader and the followers are represented by the $\{0\}$ and $\{1,2,3, \cdots N\}$, respectively. The dynamics of the follower agent can be described by:

$$
\dot{x}_{i}(t)=\mathrm{A} x_{i}(t)+\mathrm{B} u_{i}(t), i=1,2,3, \cdots N
$$

where $\mathrm{A} \in \mathbb{R}^{n \times n}$ and $\mathrm{B} \in \mathbb{R}^{n \times m}$ are the given matrices with $\operatorname{rank}(\mathrm{B})=\mathrm{m}$, and $\mathrm{n} \geq \mathrm{m}$, $x_{i}(t) \in \mathbb{R}^{n}$ is the state of the $i$ th follower agent and $u_{i}(t) \in \mathbb{R}^{m}$ is the corresponding input.

The leader's dynamics are described by:

$$
\dot{x}_{0}(t)=\mathrm{A} x_{0}(t)+\mathrm{B} u_{0}(t)
$$

where $x_{0}(t) \in \mathbb{R}^{n}$ and $u_{i}(t) \in \mathbb{R}^{n}$ denote the state and the control input of the leader, respectively.

Assumption 1. The pair $(A, B)$ is stabilizable.

Definition 1. The desired time-varying formation configuration is specified by the vector $h(t)=$ $\left[h_{1}^{T}(t), h_{2}^{T}(t), h_{3}^{T}(t), \cdots h_{N}^{T}(t)\right]^{T} \in \mathbb{R}^{4 N}$, with $h_{i}(t)=\left[h_{i x}, h_{i v x}, h_{i y}, h_{i v y}\right]^{T}$ as a bounded piecewise differentiable function; $h_{i x}, h_{i v x}, h_{i y}$ and $h_{i v y}$ are the desired position and velocity relative 
offsets in $X$ and $Y$ directions to the leader agent. For any given initial bounded states, if the following formula is satisfied:

$$
\lim _{t \rightarrow \infty}\left(x_{i}(t)-h_{i}(t)-x_{0}(t)\right)=0, i=1,2,3, \cdots, N
$$

then we say the multi-agent system has achieved the expected time-varying formation tracking.

Remark 1. The time-varying formation is designed by the vector $h(t)$. If the vector $h(t)=0$, then the formation tracking problem become the leader-following consensus problem; in other words, the consensus problem is just a special case of the formation problem.

Remark 2. When the MAS has achieved the expected time-varying formation tracking, the position of the leader may lie inside or outside the formation, which is specified by the $h(t)$. For example, when $\lim _{t \rightarrow \infty} \sum_{i=1}^{N} h_{i}(t)=0$, according to the formula (3), we can obtain $\lim _{t \rightarrow \infty} \frac{\sum_{i=1}^{N} x_{i}(t)}{N}-x_{0}(t)=0$, which means that the position of the leader is in the center of the time-varying formation.

\section{Main Results}

\subsection{Leader-Following Tracking Control under Fixed Topology}

In this section, consider an MAS where the communication topology is fixed. We will propose a distributed adaptive tracking control protocol to make the above system stable. Before that, following assumption should be satisfied.

Assumption 2. The node 1 which represents the leader agent is a globally reachable node in the undirected graph $\bar{G}$.

For a traditional distributed static consensus protocol to make the MAS achieve consensus, the input of each agent is:

$$
u_{i}=c \mathrm{~K} \sum_{i=1}^{N} a_{i j}\left(x_{i}-x_{j}\right), i=1,2,3 \cdots N
$$

where $c>0$ is the coupling weight between the two neighbor agents, $\mathrm{K} \in \mathbb{R}^{m \times n}$ is the feedback gain matrix and $a_{i j}$ is the entry of the adjacency matrix.

Lemma 3. [19] Supposing that the graph $G$ is connected, the $N$ agents could achieve consensus (i.e., $\lim _{t \rightarrow \infty}\left\|x_{i}-x_{j}\right\|=0, \forall i, j=1,2,3, \cdots N$ ) under the protocol (4) with $K=-B^{T} P^{-1}$ and the coupling weight $c>\frac{1}{\lambda_{2}}$, where $\lambda_{2}$ is the smallest nonzero eigenvalue of the Laplacian matrix L, and $P>0$ is the solution of the following linear matrix inequality (LMI):

$$
A^{T} P+P A-2 B B^{T}<0
$$

The conditions for the multi-agent system to achieve consensus have been given in Lemma 3. As one of the consensus conditions, the coupling weight c should be greater than or equal to $\frac{1}{\lambda_{2}}$. Hence, if we want to design the consensus protocol by the method above, the value of $\lambda_{2}$ is necessary, which means every agent should obtain the entire communication graph to compute the Laplacian matrix and its smallest nonzero eigenvalue. Therefore, the protocol above given in Lemma 3 cannot be implemented by each agent in a fully distributed fashion. On the other hand, in some extreme conditions (e.g., the sensors or the communicators malfunction), the communication between the neighbor agents may be interrupted, and the entire communication topology will also change, as well as the smallest nonzero eigenvalue $\lambda_{2}$. The multi-agent system would be unstable if we still design the consensus protocol by Equation (4).

In order to avoid these limitations, we propose the following distributed consensus protocol with an adaptive time-varying coupling weight $c(t)$, which could be adjusted by 
the information from the neighbor agents to drive the agent system to achieve the desired time-varying formation.

$$
\begin{gathered}
u_{i}(t)=K\left[\begin{array}{c}
\sum_{j \in N_{i}} a_{i j} c_{i j}(t)\left(x_{i}(t)-h_{i}(t)-\left(x_{j}(t)-h_{j}(t)\right)\right) \\
+b_{i} c_{i}(t)\left(x_{i}(t)-h_{i}(t)-x_{0}(t)\right)
\end{array}\right]+v_{i}(t) \\
\dot{c}_{i j}(t)=a_{i j}\left(x_{i}(t)-h_{i}(t)-\left(x_{j}(t)-h_{j}(t)\right)\right)^{T} \Gamma\left(x_{i}(t)-h_{i}(t)-\left(x_{j}(t)-h_{j}(t)\right)\right) \\
\dot{c}_{i}(t)=b_{i}\left(x_{i}(t)-h_{i}(t)-x_{0}(t)\right)^{T} \Gamma\left(x_{i}(t)-h_{i}(t)-x_{0}(t)\right)
\end{gathered}
$$

where $c_{i j}(t)$ is the time-varying coupling weight between the agent $i$ and agent $j$; because the communication topology is an undirected graph, we can obtain $c_{i j}(t)=c_{j i}(t) . c_{i}(t)$ denotes the corresponding weight between the agent $i$ and the leader agent. Symbol $v_{i}(t)$ represents the compensational input, which is used to expand the feasible formation set. We will ascertain the compensational input later. Matrix $K \in \mathbb{R}^{m \times n}$ is the feedback gain, and $\Gamma$ is an $n \times n$ dimension square matrix.

Under Assumption 1, the pair (A, B) is stabilizable, and there must exist a symmetric positive definite matrix $\mathrm{P}$, which is the solution of the following Riccati inequation:

$$
A^{T} P+P A-2 P B B^{T} P+I<0
$$

thus, we let the matrix $\mathrm{K}$ and $\Gamma$ equal to $-B^{T} P$ and $P B B^{T} P$, respectively.

In protocol (6), we notice that the compensational input $v_{i}(t)$ is still uncertain. The value of $v_{i}(t)$ plays an important role to achieve the desired time-varying formation. It is a judging condition which is used to estimate whether the expected formation could be realized. In this article, we define $v_{i}(t)$ as the following formulation [29]:

$$
A h_{i}(t)-\dot{h}_{i}(t)-B u_{0}(t)+B v_{i}(t)=0, i=1,2,3, \cdots N
$$

Theorem 1. Consider an MAS with a leader represented by (1) and (2). Suppose the Assumptions 1-2 hold and the offset vector $h_{i}(t)$ satisfies the formation tracking feasibility condition (10), then the multi-agent system could achieve the desired time-varying formation by using protocol (6) under fixed communication topology.

Proof of Theorem 1. Let $e_{i}(t)=x_{i}(t)-h_{i}(t)-x_{0}(t), i=1,2,3, \cdots N$. The $e_{i}(t)$ denotes the formation tracking error of the $i$ th agent, and $\mathrm{e}(\mathrm{t})=\left[e_{1}(t), e_{2}(t), e_{3}(t), \cdots e_{N}(t)\right]^{T}$.

$$
\begin{aligned}
\dot{x}_{i}(t) & =A x_{i}(t)+B u_{i}(t) \\
& =A x_{i}(t)+B v_{i}(t) \\
& -B B^{T} P\left[\begin{array}{c}
\sum_{j \in N_{i}} a_{i j} c_{i j}(t)\left(x_{i}(t)-h_{i}(t)-\left(x_{j}(t)-h_{j}(t)\right)\right) \\
+b_{i} c_{i}(t)\left(x_{i}(t)-h_{i}(t)-x_{0}(t)\right)
\end{array}\right]
\end{aligned}
$$

according to (10) and (11), we could obtain:

$$
\begin{aligned}
\dot{e}_{i}(t) & =\dot{x}_{i}(t)-\dot{h}_{i}(t)-\dot{x}_{0}(t) \\
& =A e_{i}(t)-B B^{T} P\left[\sum_{j \in N_{i}} a_{i j} c_{i j}(t)\left(e_{i}(t)-e_{j}(t)\right)+b_{i} c_{i}(t) e_{i}(t)\right]
\end{aligned}
$$

Consider the following Lyapunov function candidate:

$$
V(t)=\sum_{i=1}^{N} e_{i}^{T} P e_{i}+\sum_{i=1}^{N} \sum_{j \in N_{i}} \frac{\left(c_{i j}(t)-\beta\right)^{2}}{2}+\sum_{i=1}^{N} \sum_{j \in N_{i}}\left(c_{i}(t)-\beta\right)^{2}
$$


where $\beta$ is a positive constant-we will determine its value later. Then, if we evaluate the

derivative along the trajectories of Equation (12), we can obtain:

$$
\begin{aligned}
\dot{V}(t) & =2 \sum_{i=1}^{N} e_{i}^{T} P \dot{e}_{i}+\sum_{i=1}^{N} \sum_{j \in N_{i}}\left(c_{i j}(t)-\beta\right) \cdot \dot{c}_{i j}(t)+2 \sum_{i=1}^{N} \sum_{j \in N_{i}}\left(c_{i}(t)-\beta\right) \cdot \dot{c}_{i}(t) \\
& =2 \sum_{i=1}^{N} e_{i}^{T} P \dot{e}_{i}+2 \sum_{i=1}^{N} \sum_{j \in N_{i}} b_{i}\left(c_{i}(t)-\beta\right) \cdot e_{i}^{T} \Gamma e_{i}+\sum_{i=1}^{N} \sum_{j \in N_{i}} a_{i j}\left(c_{i j}(t)-\beta\right) \cdot\left(e_{i}-e_{j}\right)^{T} \Gamma\left(e_{i}-e_{j}\right) \\
& =e_{i}^{T}\left[I_{N} \otimes\left(A^{T} P+P A\right)-2 \beta(L+F) \otimes \Gamma\right] e_{i}
\end{aligned}
$$

According to Lemma 2, we can rewrite (14) as following form:

$$
\dot{V}(t)=e_{i}^{T}\left[I_{N} \otimes\left(A^{T} P+P A\right)-2 \beta H \otimes \Gamma\right] e_{i}
$$

From Lemma 2, we know that $\mathrm{H}$ is a symmetric positive definite matrix, so there must exist an orthogonal matrix $\mathrm{T} \in \mathbb{R}^{N \times N}$ such that:

$$
T H T^{T}=\operatorname{diag}\left(\lambda_{1}, \lambda_{2}, \lambda_{3}, \cdots \lambda_{N}\right)
$$

where $\lambda_{1}, \lambda_{2}, \lambda_{3}, \cdots, \lambda_{N}$ are the eigenvalues of $\mathrm{H}$ and for any $i=1,2,3, \cdots N$, we have $\lambda_{i}>0$. Let $\widetilde{e}=\left(T \otimes I_{n}\right) e$ :

$$
\dot{V}(t)=\widetilde{e}^{T}\left(I_{N} \otimes\left(A^{T} P+P A\right)-2 \beta \Lambda \otimes \Gamma\right) \widetilde{e}=\sum_{i=1}^{N} \widetilde{e}_{i}^{T}\left(A^{T} P+P A-2 \beta \lambda_{i} \Gamma\right) \widetilde{e}_{i}
$$

Because the eigenvalues $\lambda_{1}, \lambda_{2}, \lambda_{3}, \cdots, \lambda_{N}$ are non-negative, there must exist a positive number $\beta$ to make $\beta \lambda_{i}>1, i=1,2,3, \cdots, N$. We could obtain:

$$
\dot{V}(t) \leq \sum_{i=1}^{N} \widetilde{e}_{i}^{T}\left(A^{T} P+P A-2 \lambda_{\min } \Gamma\right) \widetilde{e}_{i} \leq-\sum_{i=1}^{N} \widetilde{e}_{i}^{T} \widetilde{e}_{i}=-\sum_{i=1}^{N} e_{i}^{T} e_{i}
$$

thus, for any error $e_{i} \neq 0$, that always have the derivative of the Lyapunov function $\dot{V}(t)<0$, we can say (12) is globally asymptotically stable, which means the follower agents could reach the desired formation and tack the leader or the virtual leader under the fixed topology.

In order to explain the procedure of the proposed method, we use the following block diagram (Figure 1) to illustrate it.

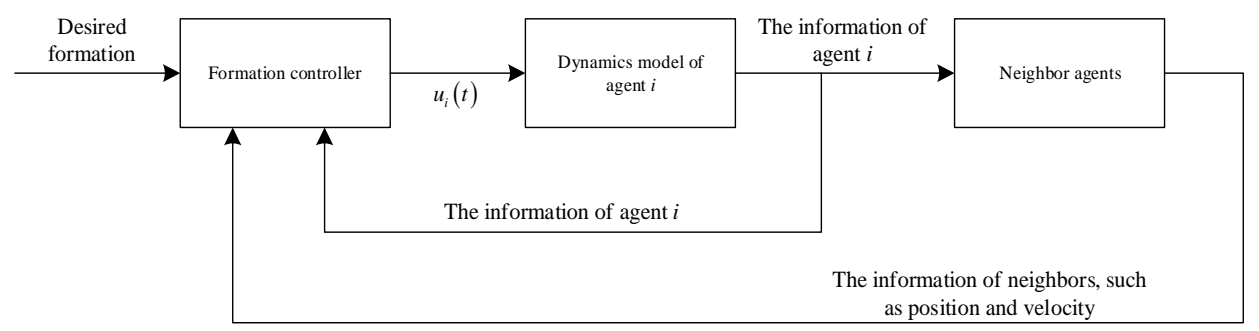

Figure 1. Block diagram of the proposed method.

First of all, the desired formation should be designed, where each agent obtains the information of itself and its neighbor agents, and the formation controller calculates the corresponding coupling weights and control input. After the control input of each agent is obtained, the information of position and speed are determined through its dynamics model. The information is sent to the formation controller and the neighbor agents. Then, the desired formation can be realized through the above cycle mode. 


\subsection{Leader-Following Tracking Control under Switching Topology}

In this section, we will focus on the formation tracking control of the MAS under switching topology, which means the graph $\bar{G}$ will change over time. Hence, while the system is running, all the possible connection situations of the swarm system should be considered. Define set $\{\bar{G}: p \in \mathcal{P}\}$ as a collection for a system connection graph, where $p$ and $\mathcal{P}$ represent the index and the index set to all possible graphs of $\bar{G}$, respectively. To describe the switching time, we introduce the switching signal $\sigma(t):[0,+\infty] \rightarrow \mathcal{P}$, at time $t$, and the connection topology among the follower agents is $\bar{G}_{\sigma(t)}$.

As the topology of the communication changes with time, the neighbors of each agent also change with it. Hence, the tracking protocol we designed is:

$$
\begin{gathered}
u_{i}(t)=K\left[\begin{array}{c}
\sum_{j \in N_{i}(t)} a_{i j}(t) c_{i j}(t)\left(x_{i}(t)-h_{i}(t)-\left(x_{j}(t)-h_{j}(t)\right)\right) \\
+b_{i}(t) c_{i}(t)\left(x_{i}(t)-h_{i}(t)-x_{0}(t)\right)
\end{array}\right]+v_{i}(t) \\
\dot{c}_{i j}(t)=a_{i j}(t)\left(x_{i}(t)-h_{i}(t)-\left(x_{j}(t)-h_{j}(t)\right)\right)^{T} \Gamma\left(x_{i}(t)-h_{i}(t)-\left(x_{j}(t)-\mathrm{h}_{j}(t)\right)\right) \\
\dot{c}_{i}(t)=b_{i}(t)\left(x_{i}(t)-h_{i}(t)-x_{0}(t)\right)^{T} \Gamma\left(x_{i}(t)-h_{i}(t)-x_{0}(t)\right)
\end{gathered}
$$

where $a_{i j}(t)$ and $b_{i j}(t)$ are the elements of the adjacency matrix and leader adjacency matrix. Because the topology is time-varying, the two matrixes above also change over time, and we denote them by $A_{\sigma(t)}$ and $F_{\sigma(t)}$, respectively.

The tracking error $e_{i}(t)$ under switching topology could be written as:

$$
\begin{aligned}
e_{i}(t) & =x_{i}(t)-h_{i}(t)-x_{0}(t) \\
& =A e_{i}(t)-B B^{T} P\left[\sum_{j \in N_{i}(t)} a_{i j}(t) c_{i j}(t)\left(e_{i}(t)-e_{j}(t)\right)+b_{i}(t) c_{i}(t) e_{i}(t)\right]
\end{aligned}
$$

Consider a series of time intervals $\left[t_{m}, t_{m+1}\right], \mathrm{m} \in \mathbb{N}$, which are infinitely bounded and continuous. In each time interval $\left[t_{m}, t_{m+1}\right]$, we divide it into several subintervals, and there is no overlap among them. To make the problem clear, use the following formula to describe each of them:

$$
\left[t_{m}^{0}, t_{m}^{1}\right), \cdots,\left[t_{m}^{j}, t_{m}^{j+1}\right), \cdots,\left[t_{m}^{n_{k}-1}, t_{m}^{n_{k}}\right), t_{m}^{0}=t_{m}, t_{m}^{n_{k}}=t_{m+1}
$$

where $t_{m}^{j+1}-t_{m}^{j} \geq \tau, \tau>0$ is a given constant, $\mathrm{n} \in \mathbb{N}^{+}$. In every subinterval the communication topology of MAS does not change.

Definition 2. The union of a collection of graphs is a graph whose vertex and edge sets are the unions of the vertex and edge sets of the graphs in the collection. We say that such a collection is jointly connected if the union of its members is a connected graph. The graphs are said to be jointly connected across the time interval $[t, t+T], T>0$, if the union of graphs $\left\{\bar{G}_{\sigma(s)}: s \in[t, t+T]\right\}$ is jointly connected.

Assumption 3. For each time interval $\left[t_{m}, t_{m+1}\right] m=0,1,2 \cdots$, the topology graphs are jointly connected for (1) and (2).

Before the stability analysis of the MAS, we should label the eigenvalues of the matrix $H_{\sigma(t)}=L_{\sigma(t)}+F_{\sigma(t)}$. Specific labeling methods are described in detail in [30]; we will explain the method briefly in the following paragraphs.

First, find all the independent components of each subgraph $G_{\sigma(t)}$ and mark them with the following symbols $S_{\sigma(t)}^{1}, S_{\sigma(t)}^{2}, \cdots S_{\sigma(t)}^{r}, 1 \leq \mathrm{r} \leq \mathrm{n}$. Rewrite the Laplacian matrix of $\bar{G}_{p}$ in the form $L_{p}=\operatorname{diag}\left(L_{\sigma(t)}^{1}, L_{\sigma(t)}^{2}, \cdots, L_{\sigma(t)}^{r}\right) ; L_{\sigma(t)}^{i} \in \mathbb{R}^{t_{\sigma(t)}^{i} \times t_{\sigma(t)}^{i}}\left(t_{\sigma(t)}^{i}=\left|\mathcal{V}\left(S_{\sigma(t)}^{i}\right)\right|\right)$ 
is the counterpart of component $S_{\sigma(t)}^{i}$, where $\mathcal{V}\left(S_{\sigma(t)}^{i}\right)=\left\{v_{1}, v_{2}, \cdots v_{t_{\sigma(t)}^{i}}\right\}$, which is the subset of $\{1,2, \cdots, n\}$ and $v_{1}<v_{2}<\cdots<v_{t_{\sigma(t)}^{i}}$.

Arrange the eigenvalues of the matrix $H_{\sigma(t)}^{i}$ in order from small to large, $\delta_{\sigma(t), 1}^{i}<\delta_{\sigma(t), 2}^{i}<$ $\cdots<\delta_{\sigma(t), t_{\sigma(t)}^{i}}^{i}$ and label them $\lambda_{\sigma(t)}^{v_{1}}\left(\leftrightarrow \delta_{\sigma(t), 1}^{i}\right), \lambda_{\sigma(t)}^{v_{2}}\left(\leftrightarrow \delta_{\sigma(t), 2}^{i}\right), \cdots \lambda_{\sigma(t)}^{v_{t \sigma}}\left(\leftrightarrow \delta_{\sigma(t), t_{\sigma(t)}^{i}}^{i}\right)$ The eigenvalue of $H_{\sigma(t)}$ is obtained by combining the eigenvalues of matrix $H_{\sigma(t)}^{1}, H_{\sigma(t)}^{2}, \cdots H_{\sigma(t)}^{r}$ and labeling all of them.

Example 1. To illustrate the rules, consider a communication graph $\bar{G}_{p}$ in Figure 2. There are three components in $G_{p}$, which are $S_{p}^{1}, S_{p}^{2}$ and $S_{p}^{3}$, respectively. According to the connection situation, the matrixes $H_{p}^{1}, H_{p}^{2}$ and $H_{p}^{3}$ are:

$$
\begin{aligned}
& H_{p}^{1}=\left[\begin{array}{ll}
1 & -1 \\
-1 & 1
\end{array}\right]+\left[\begin{array}{ll}
0 & 0 \\
0 & 0
\end{array}\right]=\left[\begin{array}{ll}
1 & -1 \\
-1 & 1
\end{array}\right] \\
& H_{p}^{2}=\left[\begin{array}{ll}
1 & -1 \\
-1 & 1
\end{array}\right]+\left[\begin{array}{ll}
1 & 0 \\
0 & 0
\end{array}\right]=\left[\begin{array}{ll}
2 & -1 \\
-1 & 1
\end{array}\right] \\
& H_{p}^{3}=[0]+[0]=[0]
\end{aligned}
$$

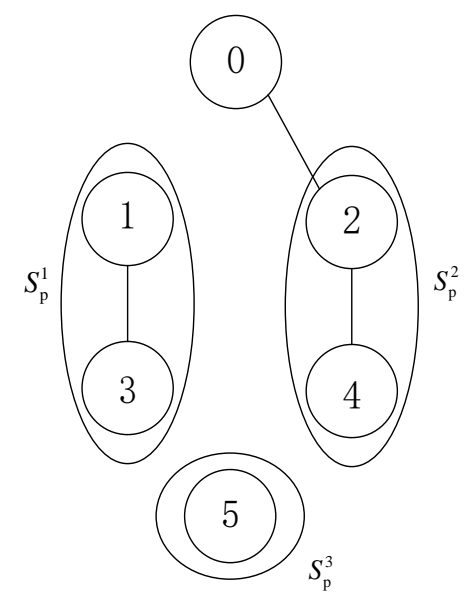

Figure 2. A graph to illustrate the labeling rules.

The eigenvalues of $H_{p}^{1}$ are 0 and 2 , then we label them as $\lambda_{p}^{1}(\leftrightarrow 0)$ and $\lambda_{p}^{3}(\leftrightarrow 2)$ by the method above. The vertices set $\mathcal{V}\left(S_{p}^{1}\right)=\{1,3\}$. The eigenvalues of $H_{p}^{2}$ are 0.382 and 2.618 , then we label them as $\lambda_{p}^{2}(\leftrightarrow 0.382)$ and $\lambda_{p}^{4}(\leftrightarrow 2.618)$. The corresponding vertices set $\mathcal{V}\left(S_{p}^{2}\right)=\{2,4\}$. As for $H_{p}^{3}$, obviously the eigenvalue and vertices set are $\lambda_{p}^{5}(\leftrightarrow 0)$ and $\mathcal{V}\left(S_{p}^{3}\right)=\{5\}$, respectively (in a sense, $H_{p}^{3}$ can be seen as a $1 \times 1$ matrix).

Therefore, all of the eigenvalues of $H_{p}$ have been labeled as $\lambda_{p}^{1}(\leftrightarrow 0), \lambda_{p}^{2}(\leftrightarrow 0.382)$, $\lambda_{p}^{3}(\leftrightarrow 2), \lambda_{p}^{4}(\leftrightarrow 2.618)$ and $\lambda_{p}^{5}(\leftrightarrow 2.618)$.

According to the above labeling method, for $\forall$, there are always $\mathrm{n}$ eigenvalue sequences of $H_{p}$ as: $\left\{\lambda_{p}^{1}, \lambda_{p}^{2}, \cdots, \lambda_{p}^{n}\right\}$. We define set

$$
1(p)=\left\{\begin{array}{c}
k, \text { correspanding eigenvalue } \lambda_{p}^{k} \text { is nonzero, } \\
k=1,2, \cdots
\end{array}\right\}
$$


Lemma 4. If the graphs are jointly connected in the time interval $\left[t_{m}, t_{m+1}\right)$, then we have:

$$
\underset{t \in\left[t_{m}, t_{m+1}\right)}{\cup} l(\sigma(t))=\{1,2, \cdots, N\}
$$

Theorem 2. If the multi-agent systems (1) and (2) satisfy Assumption 1, and the interaction topology which is described by the switching signal $\sigma(t)$ satisfies Assumption 3, there exists a symmetric positive definite matrix $P$, which is the solution of the following algebraic Riccati inequation:

$$
A^{T} P+P A-2 P B B^{T} P+\theta I<0
$$

where $\theta$ is a positive number. Let feedback gain matrix $K=B^{T} P$, then for any initial conditions, the swarm systems could track the leader agent and achieve the desired formation under protocol (19).

Proof of Theorem 2. Consider the following Lyapunov function candidate:

$$
V_{2}(t)=\sum_{i=1}^{N} e_{i}^{T} P e_{i}+\sum_{i=1}^{N} \sum_{j \in N_{i}(t)} \frac{\left(c_{i j}(t)-\beta\right)^{2}}{2}+\sum_{i=1}^{N} \sum_{j \in N_{i}(t)}\left(c_{i}(t)-\beta\right)^{2}
$$

where $\beta$ is a positive constant to be determined later. The Lyapunov function $V_{2}(t)$ is continuous and differentiable at any time except the switching instants. Then, we take the derivative of the function along the solution of (22) as:

$$
\begin{aligned}
\dot{V}_{2}(t) & =2 \sum_{i=1}^{N} e_{i}^{T} P \dot{e}_{i}+\sum_{i=1}^{N} \sum_{j \in N_{i}(t)} a_{i j}(t)\left(c_{i j}(t)-\beta\right) \cdot\left(e_{i}-e_{j}\right)^{T} \Gamma\left(e_{i}-e_{j}\right) \\
& +2 \sum_{i=1}^{N} \sum_{j \in N_{i}(t)} b_{i}(t)\left(c_{i}(t)-\beta\right) \cdot e_{i}^{T} \Gamma e_{i} \\
& =e_{i}^{T}\left[I_{N} \otimes\left(A^{T} P+P A\right)-2 \beta H_{\sigma(t)} \otimes \Gamma\right] e_{i} \#
\end{aligned}
$$

Matrix $H_{\sigma(t)}$ is symmetric, and we can always find an orthogonal matrix $T_{\sigma(t)}$ at any non-switching instants to translate the $H_{\sigma(t)}$ into diagonal forms.

$$
T_{p} H_{\sigma(t)} T_{p}^{T}=\Lambda_{\sigma(t)}=\operatorname{diag}\left(\lambda_{\sigma(t)}^{\pi_{p}(1)}, \lambda_{\sigma(t)}^{\pi_{p}(2)}, \cdots, \lambda_{\sigma(t)}^{\pi_{p}(n)}\right)
$$

where $\pi_{p}$ is some sort of permutation for the set $\{1,2, \cdots n\}$.

Let $\widetilde{e}=\left(T_{\sigma(t)} \otimes I_{n}\right) e$, we can obtain:

$$
\begin{aligned}
\dot{V}_{2}(t) & =\widetilde{e}^{T}\left[I_{n} \otimes\left(A^{T} P+P A\right)-2 \beta \Lambda_{\sigma(t)} \otimes \Gamma\right] \widetilde{e} \\
& \leq \sum_{i \in l(p)} \widetilde{e}_{i}^{T}\left[I_{n} \otimes\left(A^{T} P+P A\right)-2 \beta \lambda_{i} \otimes \Gamma\right] \widetilde{e}_{i} \\
& \leq-\sum_{i \in l(p)} \widetilde{e}_{i}^{T} \widetilde{e}_{i} \leq 0
\end{aligned}
$$

Because the coupling weights $c_{i j}(t)$ and $c_{i}(t)$ are incremental, the positive constant $\beta$ could be large enough to make $\beta \lambda_{i}>1$, for any $i \in 1(p)$.

From the above we can see that $\dot{V}_{2}(t) \leq 0$, which means $\lim _{t \rightarrow \infty} V_{2}(t)$ exists.

According to the Cauchy's convergence criteria, an infinite sequence $V_{2}\left(t_{i}\right), \mathrm{i}=0,1,2, \cdots$, for any $\mu>0$, there always exists a positive number $M_{\mu}$, such that for any $\mathrm{m}>M_{\mu}$ :

$$
\left|\int_{t_{m}}^{t_{m+1}} \dot{V}_{2}(t) d t\right|<\mu
$$


Rewrite the above inequation as the sum of multiple integrals:

$$
\int_{t_{m}^{0}}^{t_{m}^{1}}\left|\dot{V}_{2}(t)\right| d t+\int_{t_{m}^{1}}^{t_{m}^{2}}\left|\dot{V}_{2}(t)\right| d t+\cdots+\int_{t_{m}^{n_{k}-1}}^{t_{k}^{n_{k}}}\left|\dot{V}_{2}(t)\right| d t<\mu
$$

For each subitem:

$$
\int_{t_{m}^{j}}^{t_{m}^{j+1}}\left|\dot{V}_{2}(t)\right| d t=\int_{t_{m}^{j}}^{t_{m}^{j+1}}\left(-\dot{V}_{2}(t)\right) d t \geq \int_{t_{m}^{j}}^{t_{m}^{j+1}} \sum_{i \in l\left(\sigma\left(t_{m}^{j}\right)\right)} \widetilde{e}_{i}^{T} \widetilde{e}_{i} d t \geq \int_{t_{m}^{j}}^{\tau} \sum_{i \in l\left(\sigma\left(t_{m}^{j}\right)\right)} \widetilde{e}_{i}^{T} \widetilde{e}_{i} d t
$$

Thus, we have:

$$
\begin{aligned}
& \int_{t_{m}^{0}}^{t_{m}^{0}+\tau} \sum_{i \in l\left(\sigma\left(t_{m}^{0}\right)\right)} \widetilde{e}_{i}^{T}(s) \widetilde{e}_{i}(s) d s+\int_{t_{m}^{1}}^{t_{m}^{1}+\tau} \sum_{i \in l\left(\sigma\left(t_{m}^{1}\right)\right)} \widetilde{e}_{i}^{T}(s) \widetilde{e}_{i}(s) d s \\
& +\cdots+\int_{t_{m}^{n_{k-1}}}^{n_{k_{k}-1}-1}+\tau \sum_{i \in l\left(\sigma\left(t_{m}^{n_{k-1}}\right)\right)} \widetilde{e}_{i}^{T}(s) \widetilde{e}_{i}(s) d s<\mu
\end{aligned}
$$

There are finite sequences between the time interval $\left[t_{m}, t_{m+1}\right]$, so the value of the positive integer $n_{k}$ is also finite. Thus, for $\mathrm{m}>M_{\mu}$ :

$\lim _{t \rightarrow \infty} \int_{t}^{t+\tau}\left[\sum_{i \in l\left(\sigma\left(t_{k}^{0}\right)\right)} e_{i}^{T}(s) e_{i}(s)+\sum_{i \in l\left(\sigma\left(t_{k}^{1}\right)\right)} e_{i}^{T}(s) e_{i}(s)+\cdots+\sum_{i \in l\left(\sigma\left(t_{k}^{n_{k}-1}\right)\right)} e_{i}^{T}(s) e_{i}(s)\right] d s=0$

According to Lemma 4, when the system topology graphs are jointly connected across the time interval $\left[t_{m}, t_{m+1}\right)$, we have $\cup_{t \in\left[t_{m}, t_{m+1}\right)} l(\sigma(t))=\{1,2, \cdots, N\}$, and (32) could be rewritten as:

$$
\lim _{t \rightarrow \infty} \int_{t}^{t+\tau}\left[\sum_{i=1}^{n} a_{i} \widetilde{e}_{i}^{T}(s) \widetilde{e}_{i}(s)\right] d s=0
$$

where $a_{1}, a_{2}, \cdots a_{n}$ are positive integers.

Thus, we have $\lim _{t \rightarrow \infty} \sum_{i=1}^{n} a_{i} \widetilde{e}_{i}^{T}(t) \widetilde{e}_{i}(t)=0$. It is not difficult to work out $\lim _{t \rightarrow \infty} \widetilde{e}_{i}(t)=0$, which implies that $\lim _{t \rightarrow \infty} e_{i}(t)=0$. It means that the multi-agent system could track the leader agent and achieve the desired formation with the adaptive protocol (19) under switching topology.

\section{Numerical Simulation}

In this section, we will present two numerical examples to validate the feasibility of the above theoretical results. In the first case, a multi-agent system is considered, and the follower agents are supposed to track the leader and achieve a diamond time-invariant formation under fixed interaction topology with protocol (6). In the other example, the follower agents will enclose the leader while the leader is moving under switching topology with protocol (19) above.

Case 1. Suppose there is a swarm system composed of five UAVs, which consists of four followers and one leader. The communication relationship among them is shown in Figure 3a. We assume that all UAVs are flying at the same altitude. Similar to the literature [11], "because the formation tracking discussed in this paper is mainly concerned with the positions and velocities", the dynamics of the leader and follower quadrotor UAVs in the outer loop can be approximately simplified as formulas (1) and (2). The simplified communication topology is shown in Figure 3b. A similar simplification is also applied to Case 2. 


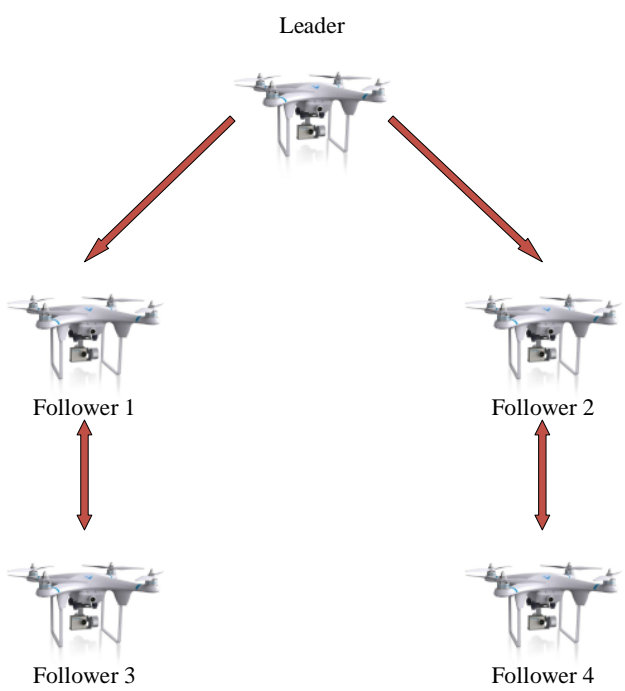

(a)

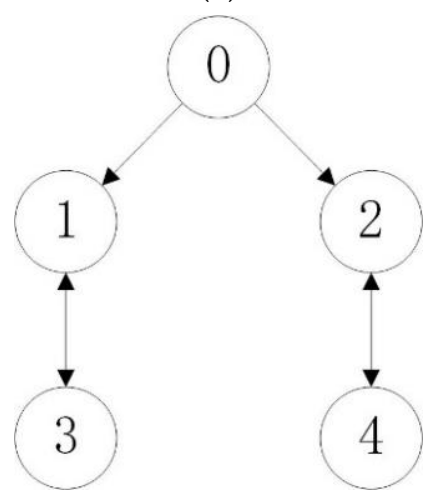

(b)

Figure 3. The interaction graph of the MASs: (a) the communication graph among the UAVs, (b) the communication graph of simplified model.

As shown in Figure $3 b$, we use " 0 " to represent the leader agent, and " $1-4$ " to represent the follower agents, respectively. The corresponding Laplacian matrix of the graph $G$ and the leader adjacency matrix $F$ of the graph $\bar{G}$ are:

$$
\mathrm{L}=\left[\begin{array}{cccc}
1 & 0 & -1 & 0 \\
0 & 1 & 0 & -1 \\
-1 & 0 & 1 & 0 \\
0 & -1 & 0 & 1
\end{array}\right] \quad \mathrm{F}=\left[\begin{array}{llll}
1 & 0 & 0 & 0 \\
0 & 1 & 0 & 0 \\
0 & 0 & 0 & 0 \\
0 & 0 & 0 & 0
\end{array}\right]
$$

The matrix $\mathrm{H}$ is:

$$
\mathrm{H}=\left[\begin{array}{cccc}
2 & 0 & -1 & 0 \\
0 & 2 & 0 & -1 \\
-1 & 0 & 1 & 0 \\
0 & -1 & 0 & 1
\end{array}\right]
$$

The dynamics of each follower agent are denoted by:

$$
\dot{x}_{i}(t)=I_{2} \otimes\left[\begin{array}{ll}
0 & 1 \\
0 & 0
\end{array}\right] x_{i}(t)+I_{2} \otimes\left[\begin{array}{l}
0 \\
1
\end{array}\right] u_{i}(t), i=1,2, \cdots N
$$

where $x_{i}(t)=\left[p_{x i}(t), v_{x i}(t), p_{y i}(t), v_{y i}(t)\right]^{T}, u_{i}(t)=\left[u_{x i}(t), u_{y i}(t)\right]^{T}$.

The dynamics of the leader are similar to the followers' and we let the leader agent move in a straight line at a uniform velocity, $\left[v_{x 0}(t), v_{y 0}(t)\right]^{T}=[1,1]^{T}$. The followers 
achieve a diamond formation while tracking the leader, which is a time-invariant formation. The formation vector $h_{i}$ is described by:

$$
\left[h_{1}, h_{2}, h_{3}, h_{4}\right]=\left[\begin{array}{cccc}
-2 & -4-\frac{2 \sqrt{3}}{3} & -6 & -4+\frac{2 \sqrt{3}}{3} \\
0 & 0 & 0 & 0 \\
-2 & -4+\frac{2 \sqrt{3}}{3} & -6 & -4-\frac{2 \sqrt{3}}{3} \\
0 & 0 & 0 & 0
\end{array}\right]
$$

According to Theorem 1, if we solve the Riccati in Equation (9), we could obtain the symmetric positive definite matrix $\mathrm{P}$ and the feedback gain matrix $\mathrm{K}$.

$$
\mathrm{P}=I_{2} \otimes\left[\begin{array}{ll}
1.1807 & 1.1441 \\
1.1441 & 2.7016
\end{array}\right] \mathrm{K}=I_{2} \otimes\left[\begin{array}{ll}
0.4370 & 1.0320
\end{array}\right]
$$

In protocol (6), an important component is the time-varying coupling weight $c_{i j}(t)$ and $c_{i}(t)$. We set the initial value of the two weights as 3 , which means

$$
c_{i j}(0)=c_{i}(0)=3
$$

Figure 4 shows the tracking error of the follower agents, as the time increases, the tracking error of the followers will approach zero at any bounded initial condition. This means the agents have achieved the desired formation.

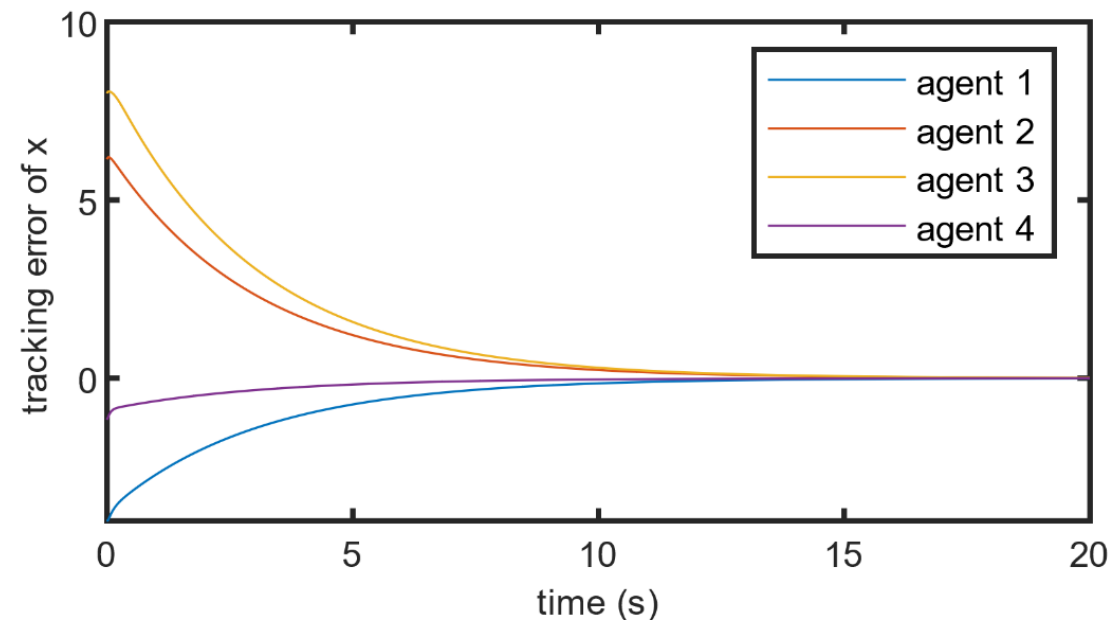

(a)

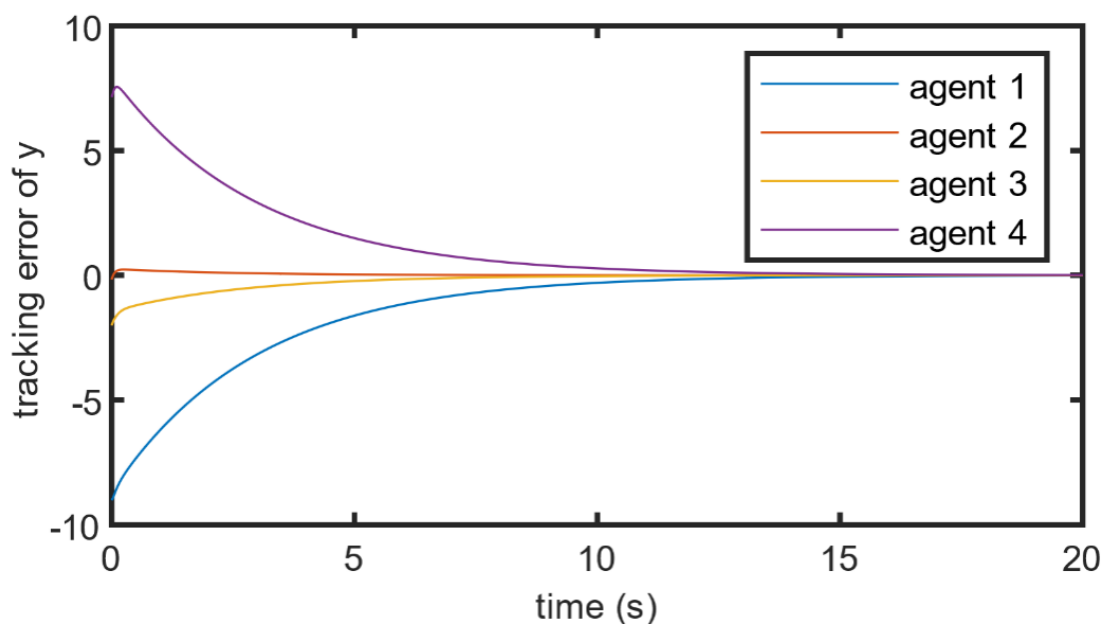

(b)

Figure 4. Tracking error of follower agents: (a) the tracking error of $x,(\mathbf{b})$ the tracking error of $y$. 
Figure 5 depicts the trajectory of the four follower agents and the leader agent. In the

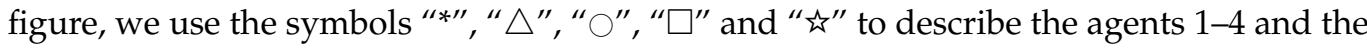
leader, respectively.

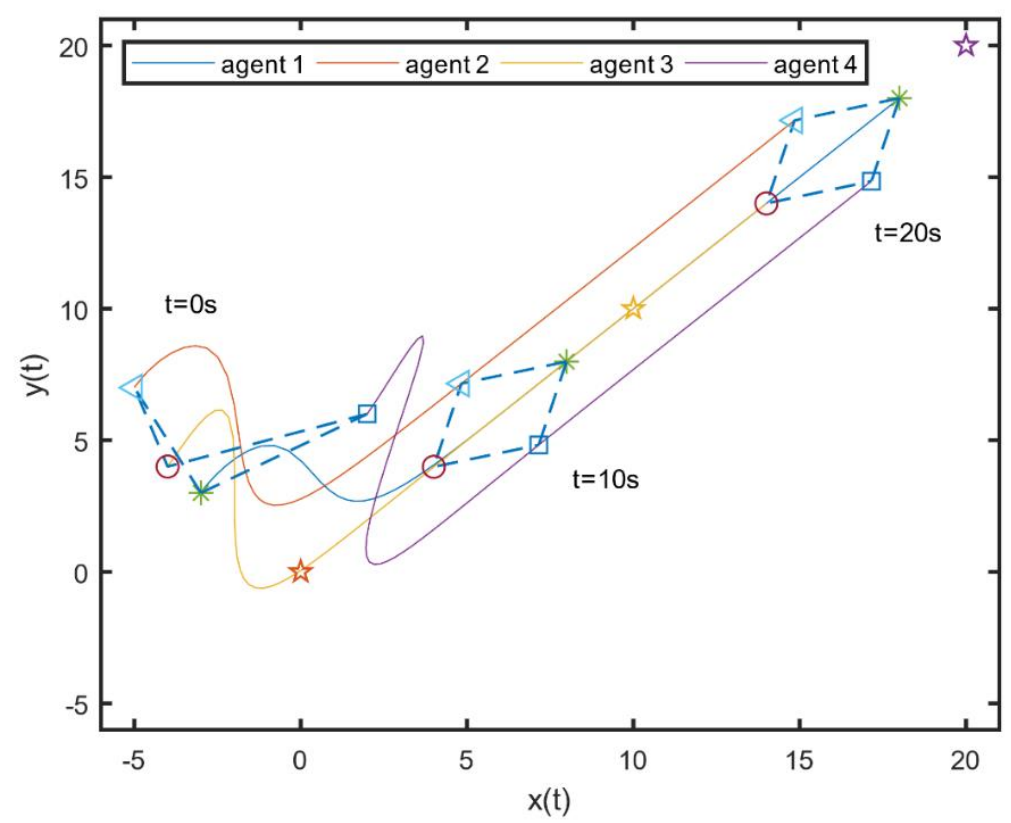

Figure 5. The trajectory of the four follower agents and the leader agent.

Case 2. Consider an MAS consisting of a leader and four follower agents with protocol (19) under switching topology. The possible interaction graphs $\left\{\bar{G}_{1}, \bar{G}_{2}, \bar{G}_{3}, \bar{G}_{4} \bar{G}_{5}, \bar{G}_{6}\right\}$ are shown in Figure 6. From Figure 6, we find out that every possible graph is nonconnected, but the union of them is jointly connected. According to Theorem 2, the agents will achieve the desired formation. The interaction graphs are switched in order of $\bar{G}_{1} \rightarrow \bar{G}_{2} \rightarrow \bar{G}_{3} \rightarrow \bar{G}_{4} \rightarrow \bar{G}_{5} \rightarrow \bar{G}_{6} \rightarrow \bar{G}_{1} \cdots$. The active time of the adjacent graphs is $1 / 3$ s, which means the switching period of the whole system is two seconds. The switching signal $\sigma(t)$ is shown in Figure 7.

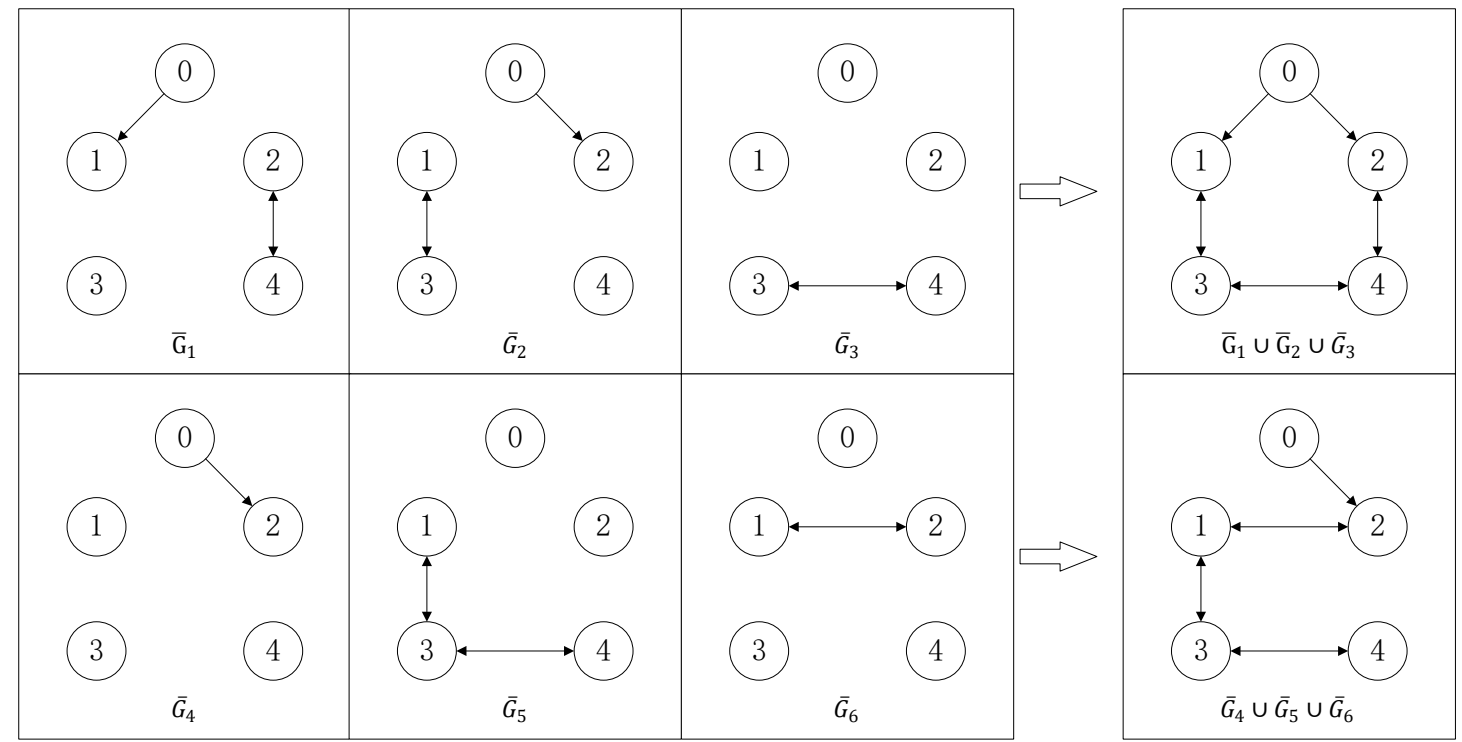

Figure 6. Possible communication topologies among the leader and the agents. 


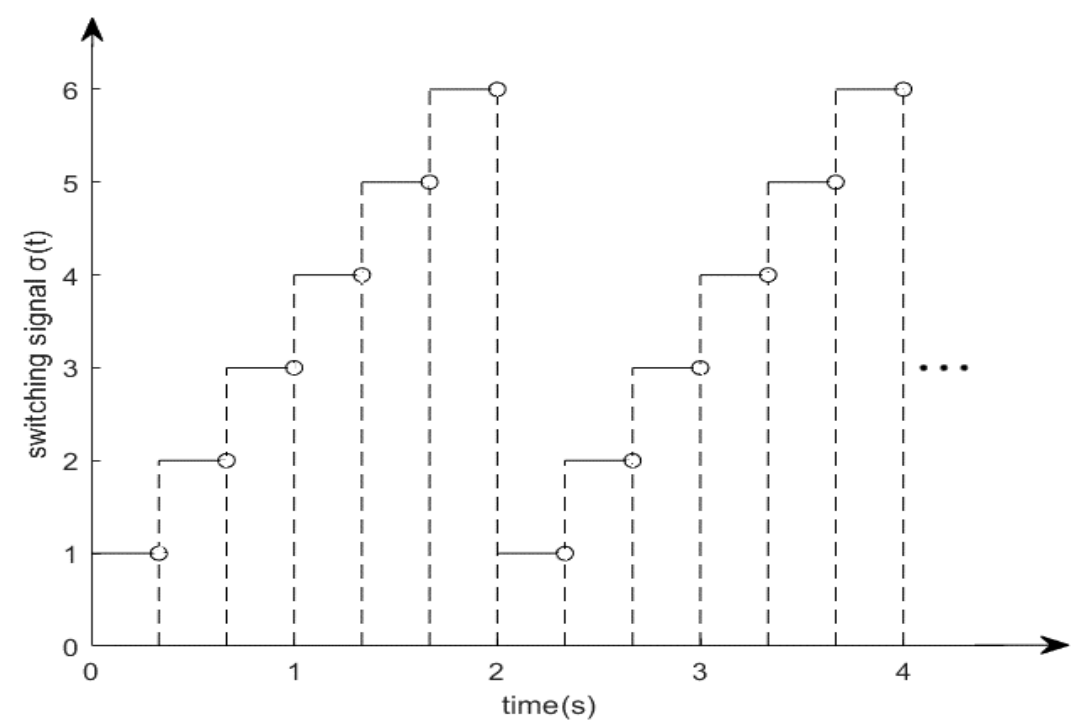

Figure 7. Switching signal function $\sigma(t)$.

First, we should obtain the corresponding solution of Equation (23).

$$
\mathrm{P}=I_{2} \otimes\left[\begin{array}{ll}
1.5120 & 2.0362 \\
2.0362 & 6.1574
\end{array}\right] \mathrm{K}=I_{2} \otimes\left[\begin{array}{ll}
0.2456 & 0.7426
\end{array}\right]
$$

In this case, we let the leader agent be stationary at the coordinate $(5,5)$ and the follower agents are supposed to revolve around the leader agent to achieve a time-varying circular formation. Assume the dynamical model is same as that of Case 1. The corresponding formation vector is:

$$
h_{i}(t)=\left[\begin{array}{c}
2 \cos \left(t-\frac{i-1}{2} \pi\right) \\
-2 \sin \left(t-\frac{i-1}{2} \pi\right) \\
2 \sin \left(t-\frac{i-1}{2} \pi\right) \\
2 \cos \left(t-\frac{i-1}{2} \pi\right)
\end{array}\right] i=1,2,3,4
$$

Obviously, the formation vector $h_{i}(t) i=1,2,3,4$ above satisfies the situation which is mentioned in Remark 2. This means the followers will enclose the leader and achieve a time-varying circle around it.

Figure 8 displays the four followers' tracking error of the $x$-axis and $y$-axis under switching topology. From the figure, we can find out that as time goes on, the error converges to zero asymptotically. Figure 9 shows the velocity trajectories of the four followers in numerical simulation. The initial condition of the velocities is zero, and after a period of adjustment, all of them tend to the speed which we desired. 


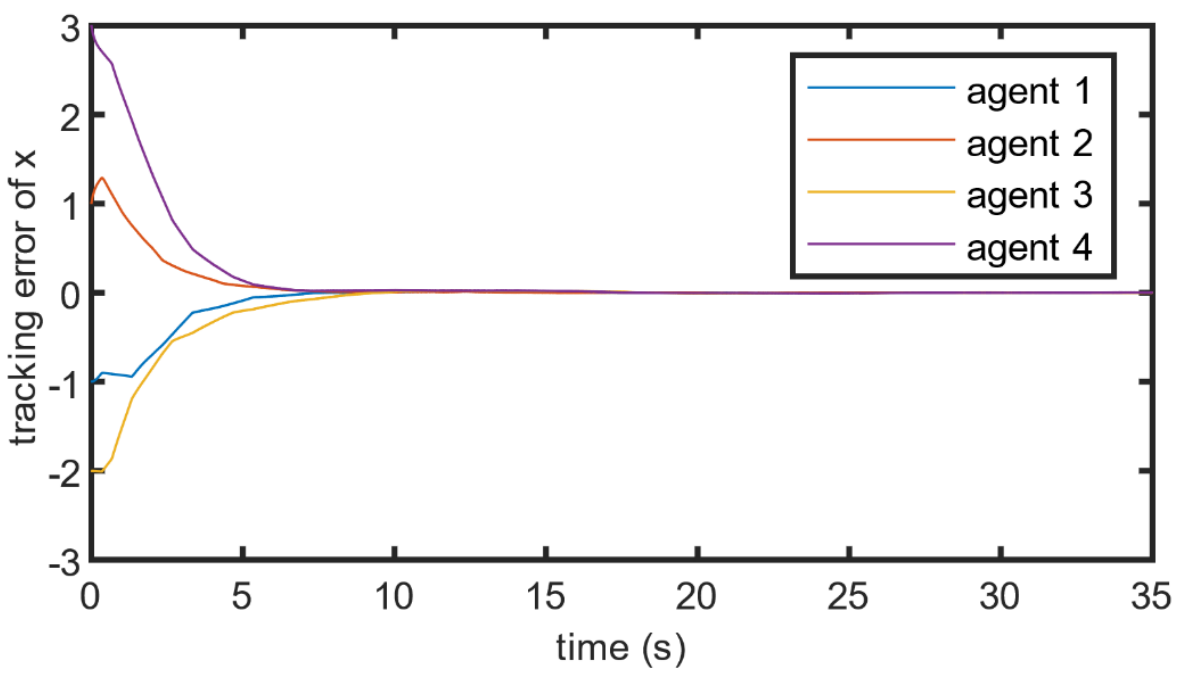

(a)

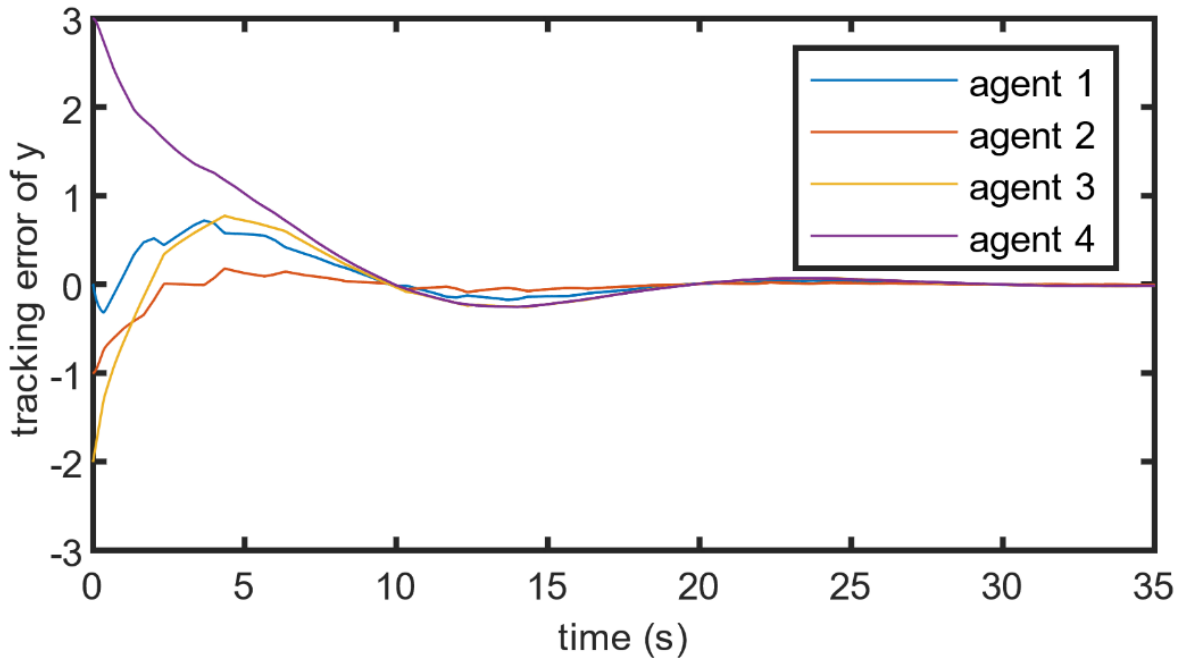

(b)

Figure 8. Tracking error under switching topology: (a) tracking error of $x,(\mathbf{b})$ tracking error of $y$.

Figure 10 depicts the snapshot of relative position at the different instants $0,3,15$ and 35 s. In every snapshot, we use the symbols “*”, “ $\triangle$ ", “ $\bigcirc ”, ~ “ \square ”$ and “㧒” to describe the agents $1-4$ and the leader, respectively.

Next, we will introduce the definition of formation tracking error, which is denoted by a Euclidean norm as $\varepsilon=\max _{\forall i, j \in\{1,2,3, \cdots, N\}}\left\|e_{i}(t)-e_{j}(t)\right\|$. Figure 11 displays the comparison results of the formation tracking error between the proposed method and the schema which has the constant coupling weights. From the figure, we could clearly find out that the formation has less fluctuations and exhibits a faster convergence rate with the adaptive strategy. 


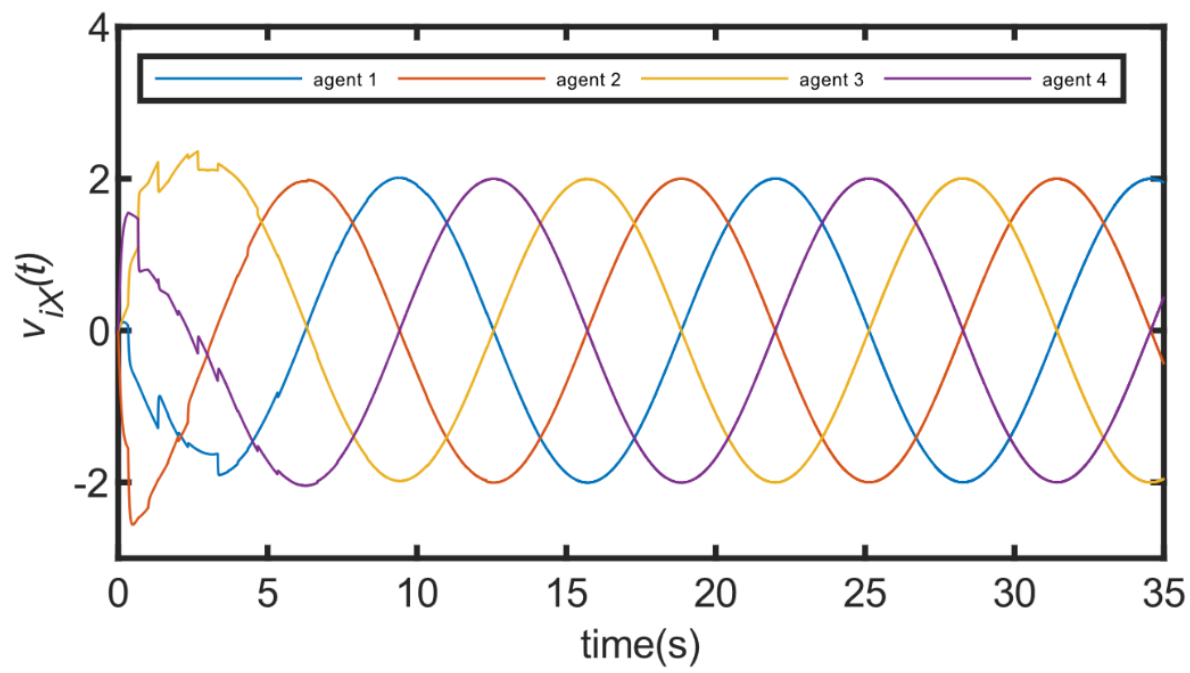

(a)

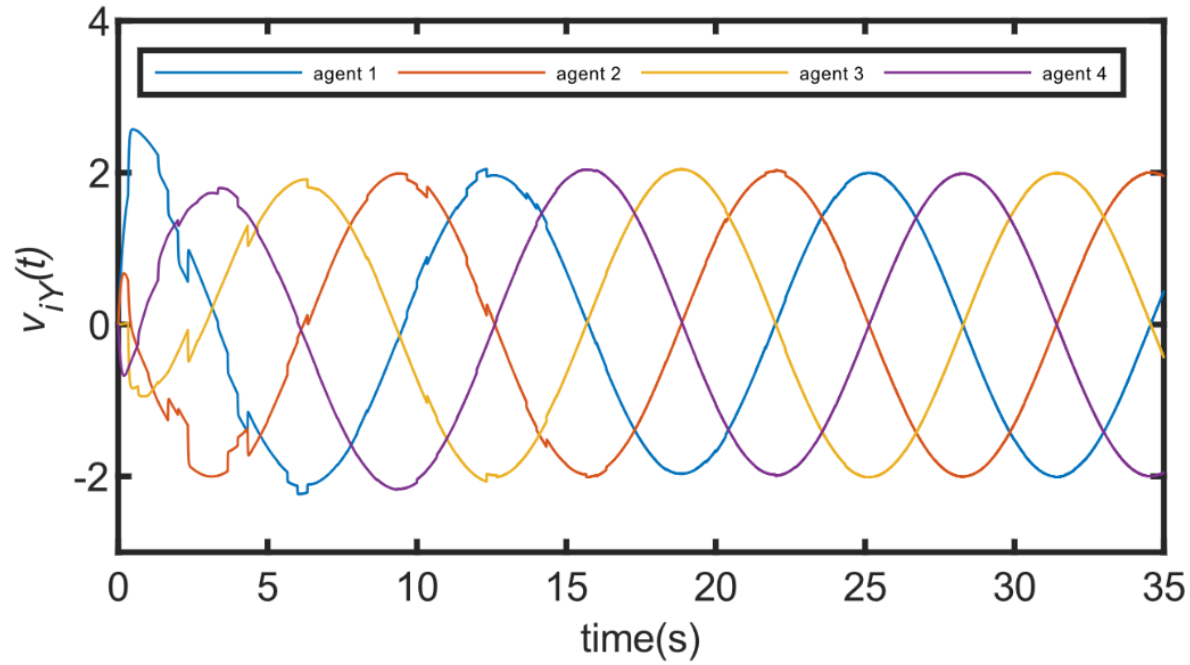

(b)

Figure 9. Velocity trajectories of the four followers: (a) represents velocity of $x,(\mathbf{b})$ represents velocity of $y$.

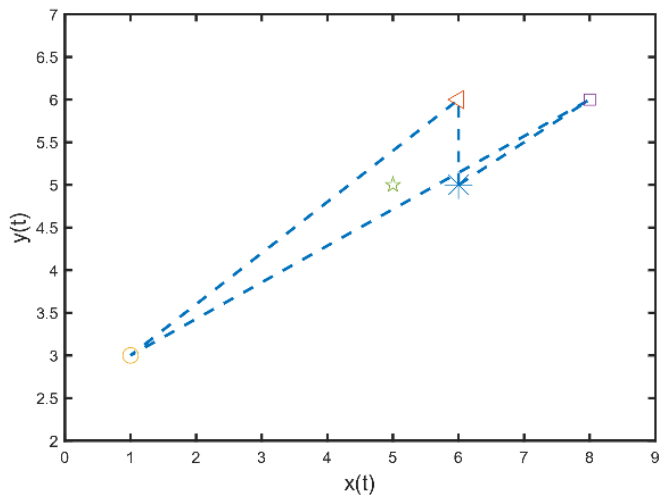

(a)

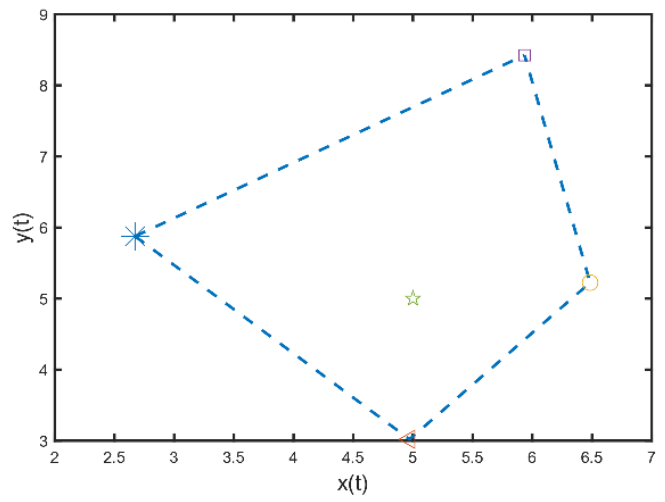

(b)

Figure 10. Cont. 


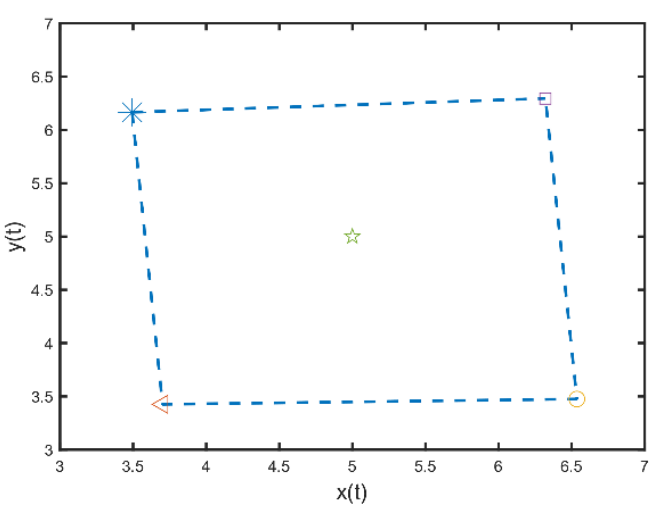

(c)

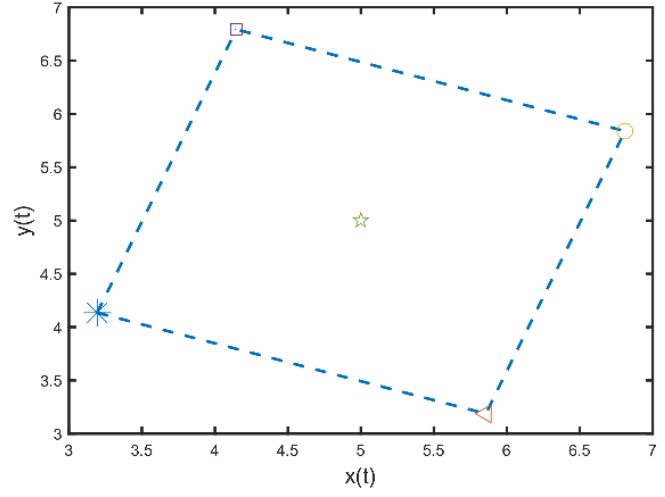

(d)

Figure 10. Relative position snapshots of agents at different instants: (a) relative position at $t=0 \mathrm{~s},(\mathbf{b})$ relative position at $\mathrm{t}$ $=3 \mathrm{~s},(\mathbf{c})$ relative position at $\mathrm{t}=15 \mathrm{~s},(\mathbf{d})$ relative position at $\mathrm{t}=35 \mathrm{~s}$.

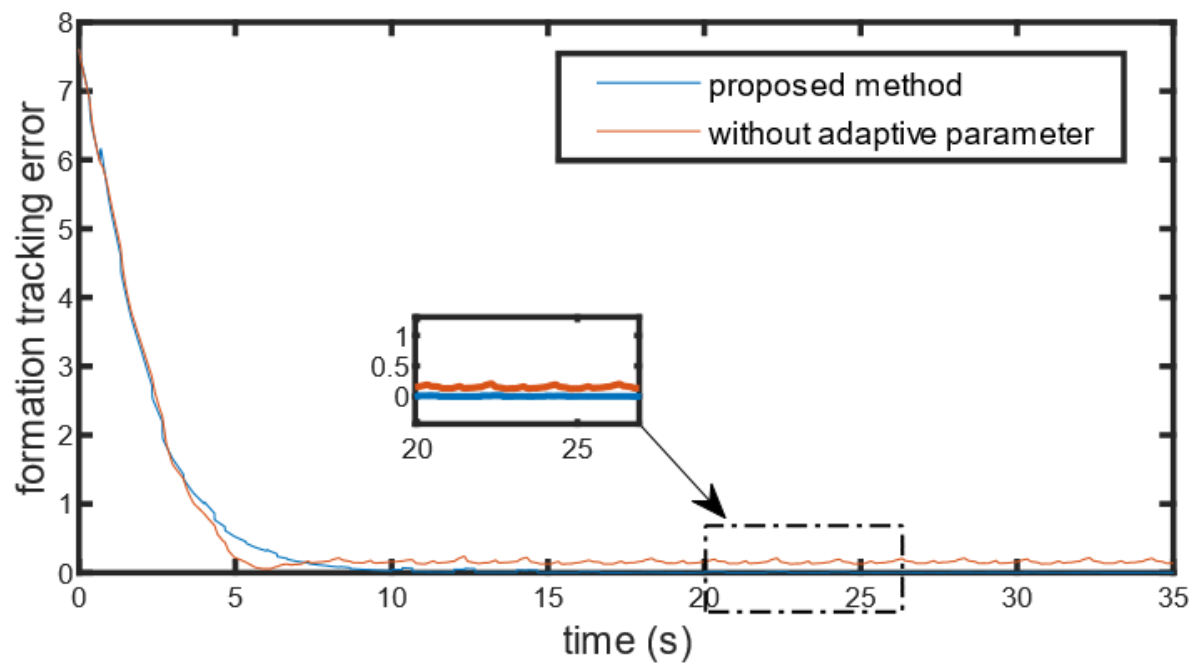

Figure 11. The comparison results between the proposed method and the schema with the constant coupling weights.

\section{Conclusions}

In this paper, the time-varying formation tracking problem for general MAS was addressed. A distributed adaptive formation controller based on Riccati inequalities was proposed to solve the above problem. The proposed controller could adjust the coupling weights by the information from the neighbor agents, which is different from the traditional distributed static controller. In addition, we also discussed the condition of achieving formation for MASs under fixed and switching topologies. The desired formation could be reached under a jointly connected graph with the proposed method. The stability was demonstrated by using Lyapunov's method. Last, two numerical simulations are given to show the efficiency of our method. The comparison experiments in the simulation demonstrated that the adaptive strategy has a positive compensating effect under a switching topology situation.

In this article, the dynamics model and formation controller were both built by MATLAB/Simulink. The experimental data which we acquired was the result of computer simulation. In the follow-up work, we would build a physical UVA platform to verify the effectiveness of the proposed algorithm.

Future research topics will extend the results to heterogeneous MAS or with a communication time delay. 
Author Contributions: Software, data curation, writing-original draft preparation, Y.Y. and T.S.; methodology, validation, Y.Y. and H.L.; writing-review and editing, T.L.; supervision, project administration, T.L. and Z.C.; funding acquisition, Y.Y. All authors have read and agreed to the published version of the manuscript.

Funding: This work is supported by the National Key R\&D Program of China (Grant No. 2017YFC0602000), the Science and Technology Development Project of Jilin Province (Grant No. 20190303061SF) and the National Natural Science Foundation of China (Grant No. 51805200).

Institutional Review Board Statement: Not applicable.

Informed Consent Statement: Not applicable.

Data Availability Statement: The data used to support the findings of this study are included within the article.

Conflicts of Interest: The authors declare no conflict of interest.

\section{References}

1. Du, S.-L.; Sun, X.-M.; Cao, M.; Wang, W. Pursuing an evader through cooperative relaying in multi-agent surveillance networks. Automatica 2017, 83, 155-161. [CrossRef]

2. Kada, B.; Khalid, M.; Shaikh, M.S. Distributed cooperative control of autonomous multi-agent UAV systems using smooth control. J. Syst. Eng. Electron. 2020, 31, 1297-1307. [CrossRef]

3. Gui, H.; Vukovich, G. Distributed almost global finite-time attitude consensus of multiple spacecraft without velocity measurements. Aerosp. Sci. Technol. 2018, 75, 284-296. [CrossRef]

4. Carli, R.; Cavone, G.; Epicoco, N.; Di Ferdinando, M.; Scarabaggio, P.; Dotoli, M. Consensus-Based Algorithms for Controlling Swarms of Unmanned Aerial Vehicles(Conference Paper). Lect. Notes Comput. Sci. 2020, 12338, 84-99.

5. Zhang, S.; Li, Z.; Wang, X. Robust H2 Consensus for Multi-Agent Systems with Parametric Uncertainties. IEEE Trans. Circuits Syst. II Express Briefs 2021. [CrossRef]

6. Dong, X.; Li, Q.; Ren, Z.; Zhong, Y. Formation-containment control for high-order linear time-invariant multi-agent systems with time delays. J. Frankl. Inst. 2015, 352, 3564-3584. [CrossRef]

7. Wang, Q.; Yu, Y.; Sun, C. Distributed event-based consensus control of multi-agent system with matching nonlinear uncertainties. Neurocomputing 2018, 272, 694-702. [CrossRef]

8. Consolini, L.; Morbidi, F.; Prattichizzo, D.; Tosques, M. Leader-follower formation control of nonholonomic mobile robots with input constraints. Automatica 2008, 44, 1343-1349. [CrossRef]

9. Abbaspour, A.; Moosavian, S.A.A.; Alipour, K. Formation control and obstacle avoidance of cooperative wheeled mobile robots. Int. J. Robot. Autom. 2015, 30, 418-428. [CrossRef]

10. Lee, G.; Chwa, D. Decentralized behavior-based formation control of multiple robots considering obstacle avoidance. Intell. Serv. Robot. 2018, 11, 127-138. [CrossRef]

11. Dong, X.; Zhou, Y.; Ren, Z.; Zhong, Y. Time-Varying Formation Tracking for Second-Order Multi-Agent Systems Subjected to Switching Topologies With Application to Quadrotor Formation Flying. IEEE Trans. Ind. Electron. 2017, 64, 5014-5024. [CrossRef]

12. Djaidja, S.; Wu, Q. Consensus of double-integrator multi-agent systems without relative states derivative under relative-state dependent measurement noises. Int. J. Syst. Sci. 2019, 50, 777-790. [CrossRef]

13. Mu, B.; Li, H.; Ding, J.; Shi, Y. Consensus in second-order multiple flying vehicles with random delays governed by a Markov chain. J. Frankl. Inst. Eng. Appl. Math. 2015, 352, 3628-3644. [CrossRef]

14. Qin, J.; Yu, C. Cluster consensus control of generic linear multi-agent systems under directed topology with acyclic partition. Automatica 2013, 49, 2898-2905. [CrossRef]

15. Ren, W. Consensus strategies for cooperative control of vehicle formations. IET Control Theory Appl. 2007, 1, 505-512. [CrossRef]

16. Seo, J.; Kim, Y.; Kim, S.; Tsourdos, A. Consensus-based reconfigurable controller design for unmanned aerial vehicle formation flight. Proc. Inst. Mech. Eng. Part G J. Aerosp. Eng. 2012, 226, 817-829. [CrossRef]

17. Dong, X.; Yu, B.; Shi, Z.; Zhong, Y. Time-Varying Formation Control for Unmanned Aerial Vehicles: Theories and Applications. IEEE Trans. Control Syst. Technol. 2015, 23, 340-348. [CrossRef]

18. Dong, X.; Xiang, J.; Han, L.; Li, Q.; Ren, Z. Distributed Time-Varying Formation Tracking Analysis and Design for Second-Order Multi-Agent Systems. J. Intell. Robot. Syst. 2017, 86, 277-289. [CrossRef]

19. Li, Z.; Ren, W.; Liu, X.; Fu, M. Consensus of Multi-Agent Systems With General Linear and Lipschitz Nonlinear Dynamics Using Distributed Adaptive Protocols. IEEE Trans. Autom. Control 2013, 58, 1786-1791. [CrossRef]

20. Chu, H.; Cai, Y.; Zhang, W. Consensus tracking for multi-agent systems with directed graph via distributed adaptive protocol. Neurocomputing 2015, 166, 8-13. [CrossRef]

21. Ding, Z.; Li, Z. Distributed adaptive consensus control of nonlinear output-feedback systems on directed graphs. Automatica 2016, 72, 46-52. [CrossRef] 
22. Zhang, Y.; Liang, H.; Ma, H.; Zhou, Q.; Yu, Z. Distributed adaptive consensus tracking control for nonlinear multi-agent systems with state constraints. Appl. Math. Comput. 2018, 326, 16-32. [CrossRef]

23. Zhang, H.; Lewis, F.L. Adaptive cooperative tracking control of higher-order nonlinear systems with unknown dynamics. Automatica 2012, 48, 1432-1439. [CrossRef]

24. Hong, Y.; Hu, J.; Gao, L. Tracking control for multi-agent consensus with an active leader and variable topology. Automatica 2006, 42, 1177-1182. [CrossRef]

25. Olfati-Saber, R.; Murray, R.M. Consensus problems in networks of agents with switching topology and time-delays. IEEE Trans. Autom. Control 2004, 49, 1520-1533. [CrossRef]

26. Ren, W.; Beard, R.W. Consensus seeking in multiagent systems under dynamically changing interaction topologies. IEEE Trans. Autom. Control 2005, 50, 655-661. [CrossRef]

27. Su, Y.; Huang, J. Stability of a Class of Linear Switching Systems with Applications to Two Consensus Problems. IEEE Trans. Autom. Control 2012, 57, 1420-1430. [CrossRef]

28. $\mathrm{Mu}$, B.X.; Shi, Y. Distributed LQR Consensus Control for Heterogeneous Multiagent Systems: Theory and Experiments. IEEEASME Trans. Mechatron. 2018, 23, 434-443. [CrossRef]

29. Deng, J.; Li, K.; Wu, S.; Wen, Y. Distributed Adaptive Time-Varying Formation Tracking Control for General Linear Multi-Agent Systems Based on Event-Triggered Strategy. IEEE Access 2020, 8, 13204-13217. [CrossRef]

30. Ni, W.; Cheng, D. Leader-following consensus of multi-agent systems under fixed and switching topologies. Syst. Control Lett. 2010, 59, 209-217. [CrossRef] 